\title{
Connections between rheumatology and oncology - discussions based on a case report
}

\author{
Laura Groseanu ${ }^{1,2}$, Mihaela Lebedenco ${ }^{3}$, Anca Ursu ${ }^{1}$, Carmen Petcu ${ }^{2}$, Ruxandra Ionescu ${ }^{1,2}$ \\ 1"Carol Davila" University of Medicine and Pharmacy, Bucharest, Romania \\ 2 "Sfanta Maria" Clinical Hospital, Bucharest, Romania \\ 3 "Coltea" Clinical Hospital, Bucharest, Romania
}

\begin{abstract}
Tumor necrosis factor (TNF) inhibitors are commonly utilized medications for the treatment of immune mediated conditions. Based on recent data from most registries there is no conclusive evidence for an increased risk of solid tumours or lymphoproliferative disease linked with biologic therapy, but on-going vigilance is required.

We present a case of ankylosing spondylitis with extraarticular manifestations with a premalignant condition treated with TNF inhibitors, which developed a basal cell cancer, was switched on secukinumab and back again on antiTNF due to severe panuveitis.
\end{abstract}

Keywords: ankylosing spondylitis, lymphoproliferative disease, antiTNF, malignancy

\section{INTRODUCTION}

Tumor necrosis factor (TNF) inhibitors are commonly utilized medications for the treatment of immune mediated conditions. Based on recent data from most registries there is no conclusive evidence for an increased risk of solid tumours or lymphoproliferative disease linked with biologic therapy, but on-going vigilance is required. There is conflicting evidence regarding the risk of skin cancers with anti-TNF therapy; patients should be advised of the need for preventative skin care, skin surveillance and prompt reporting of new persistent skin lesions. Caution should be exercised in the use of biologics in patients with previous malignancy. The timing of commencement of biologic therapy post-malignancy is not fixed and will depend on type and stage of malignancy, risk of metastasis and patient views. The effect of biologics on pre-malignant conditions remains unclear. Caution should be exercised in the use of biologics in such patients.

We present a patient with active ankylosing spondylitis and extraarticular manifestations with a monoclonal gammapathy of undetermined significance treated with TNF inhibitors, which developed also a basal cell cancer, switched on secukinumab and back again on antiTNF due to severe panuveitis.

\section{CASE REPORT}

A 67 years old male was first evaluated in Rheumatology Department of "Sfanta Maria" Clinical Hospital in august 2015 for important weight loss, polyarthritis of small joints of the hands, elbows, knees, ankles and dactilytis of $3^{\text {rd }}$ and $4^{\text {th }}$ finger of lower left limb. Detailed history revealed recurrent asymmetric polyarthritis of small and large joints, inflammatory low back pain for 20 years that the patient related to daily train travelling. Six month earlier he developed an episode of acute anterior uveitis. Physical examination revealed swelling of elbows, knees with semiflexion ankylosis, $3^{\text {rd }}$ and $4^{\text {th }}$ finger dactilytis of the lower left limb, severe limitation of spine mobility - occiput to wall index $=5 \mathrm{~cm}$, chin to sternum $=4 \mathrm{~cm}$, Schober $=3.5 \mathrm{~cm}$, finger to floor distance $=40 \mathrm{~cm}$, chest expansion $=2 \mathrm{~cm}$.

Laboratory investigations revealed: important inflammatory syndrome $(\mathrm{ESR}=8 \mathrm{~mm} / \mathrm{h}, \mathrm{CRP}=181$ $\mathrm{mg} / \mathrm{l})$, mild anemia $(10.5 \mathrm{~g} / \mathrm{dl})$, hyperproteinemia $(8.9 \mathrm{~g} / \mathrm{dl})$ with $\operatorname{IgG}=2,452 \mathrm{mg} / \mathrm{dl}$ (normal 700-1,600 
$\mathrm{mg} / \mathrm{dl})$. Pelvic Xray showed bilateral stage IV sacroiliitis; the patient was HLAB27 positive. Opthalmologic examination revealed white-yellow keratic precipitates, corneal edema and anterior synechias.

The patient was diagnose with ankylosing spondylitis with peripheral involvement and extraarticular manifestations; he was treated with NSAIDs and sulphasalazine $2 \mathrm{~g} /$ day. After discharge, he was reffered to a hematology clinic; electrophoresis confirmed hypergammaglobulinemia $(24.2 \%)$ with present free lambda chains on immunelectrophoresis. Calcium, LDH were normal, skull Xray did not show lytic lesions, no proteinuria was detected. Bone marrow biopsy revealed small plasmocytes infiltration perivascular and small lymphoid paratrabecular infiltration (6-7\%) with CD20+ lymphocytes, most of them $\operatorname{IgM}+, \kappa / \lambda$ ratio $=6 / 1$. He was diagnosed with Ig $\mathrm{M}$ monoclonal gammopathy of undetermined significance (MGUS). No specific treatment was recommended. Until approval for biological treatment was obtained, he developed 3 more uveitis episodes.

Finally, in april 2016 treatment with Golimumab was started $(E S R=132 \mathrm{~mm} / \mathrm{h}, \mathrm{CRP}=80 \mathrm{mg} / \mathrm{l}$, BAS$\mathrm{DAI}=7.3, \mathrm{ASDAS}=5.6) .6$ months later, as the response was not good enough $(\mathrm{ESR}=34 \mathrm{~mm} / \mathrm{h}, \mathrm{CRP}$ $=39 \mathrm{mg} / \mathrm{l}$, BASDAI $=3.1$, ASDAS $=3.3$ ) and he developed one more episode of uveitis, the patient was switched on iv Infliximab. The response was good (at 3 month: ESR $=10 \mathrm{~mm} / \mathrm{h}, \mathrm{CRP}=2 \mathrm{mg} / \mathrm{l}$, BASDAI $=2.6$, ASDAS $=1.8$ ) but sustained only for 9 month $(E S R=148 \mathrm{~mm} / \mathrm{h}, \mathrm{CRP}=62 \mathrm{mg} / \mathrm{l}$, BASDAI $=6.9$, ASDAS $=4.9$ ) when he was switched on Etanercept. After one month he develops another episode of uveitis and a pearly ulcerated nodule above the right orbit is noticed at physical examination. The biopsy diagnosed basal cell cancer; the tumor was excised with safety margins. AntiTNF therapy is stopped; unfortunatelly, 2 months later the disease becomes very active again, so the decision to start secukinumab was taken $(\mathrm{ESR}=124 \mathrm{~mm} / \mathrm{h}$, $\mathrm{CRP}=98 \mathrm{mg} / \mathrm{l}, \mathrm{BASDAI}=7.9, \mathrm{ASDAS}=5.4)$. The response was good and sustained for one year (ESR $=25 \mathrm{~mm} / \mathrm{h}, \mathrm{CRP}=19 \mathrm{mg} / \mathrm{l}, \mathrm{BASDAI}=2.1$, ASDAS $=1.8$ ). In september 2018, while the disease was controlled, the patient develops an episode of panuveitis of the right eye; the vision of the left eye was compromised by a central corneal leukoma and iridocorneal adhesions after recurrent uveitis episodes. Together with 2 ophtalmologists, causes of panuveitis were reviewed and excluded by specific tests: sar- coidosis, infections (herpes, Mycoplama, Toxoplasma, Toxocara, tuberculosis, Chlamydia), trauma, Behçet disease or other inflammatory systemic conditions, drugs or toxins, Vogt-Koyanagi-Harada syndrome. He was treated with pulse therapy with methylprednisolone, followed by a course of one month with oral steroids. Current data suggest that secukinumab is not associated with an increased risk of uveitis in ankylosing spondylitis patients; in an analyse of 7,355 patients with psoriatic arthritis and ankylosing spondylitis (162,269 patient-years) only 41 cases of uveitis were reported and only 1 severe case (1). Among these 7,355 patients with ankylosing spondylitis, psoriasis, psoriatic arthritis, the EAIR (exposure-adjusted incidence rates per 100 patient-years) was 0.5 (95\% CI 0.2-0.90) (1).

Still, secukinumab was stopped with disease subquent reactivation after 2 months. After obtaining dermatologist and oncologist approval, switch on Adalimumab was decided, unfortunatelly with no benefits after 3 months. The only option left was Certolizumab. After 7 month the disease is well controlled, no more episodes of uveitis, no recurrence of basal cell cancer. During biological therapy the patient was monitored by the hematologist according to specific protocols (2); no progression of MGUS was noticed; values of Ig $\mathrm{G}$ varied between 2,283 and $1,220 \mathrm{mg} / \mathrm{dl}$.

\section{CASE DISCUSSIONS}

Our concern when we decided to start TNF inhibitors was related to the relative contraindication in patients with premalignant conditions (3).

Monoclonal gammopathy of undetermined significance (MGUS) is a premalignant, clonal plasma cell disorder, characterized by the presence of a monoclonal $(\mathrm{M})$ protein, $<10 \%$ clonal plasma cells in the bone marrow, and absence of multiple myeloma or related lymphoplasmacytic malignancies. MGUS is present in $3 \%$ of the general population $\geq 50$ years old, but only $0.3 \%$ among those $<50$ years old. It is considered a requisite precursor of multiple myeloma, as well as immunoglobulin lightchain amyloidosis and Waldenström macroglobulinemia, and can be detected years before the diagnosis of these.There are 3 subtypes of MGUS: immunoglobulin M (IgM) MGUS, non-IgM MGUS, and light-chain MGUS, with distinct rate and type of progression (Table 1). 


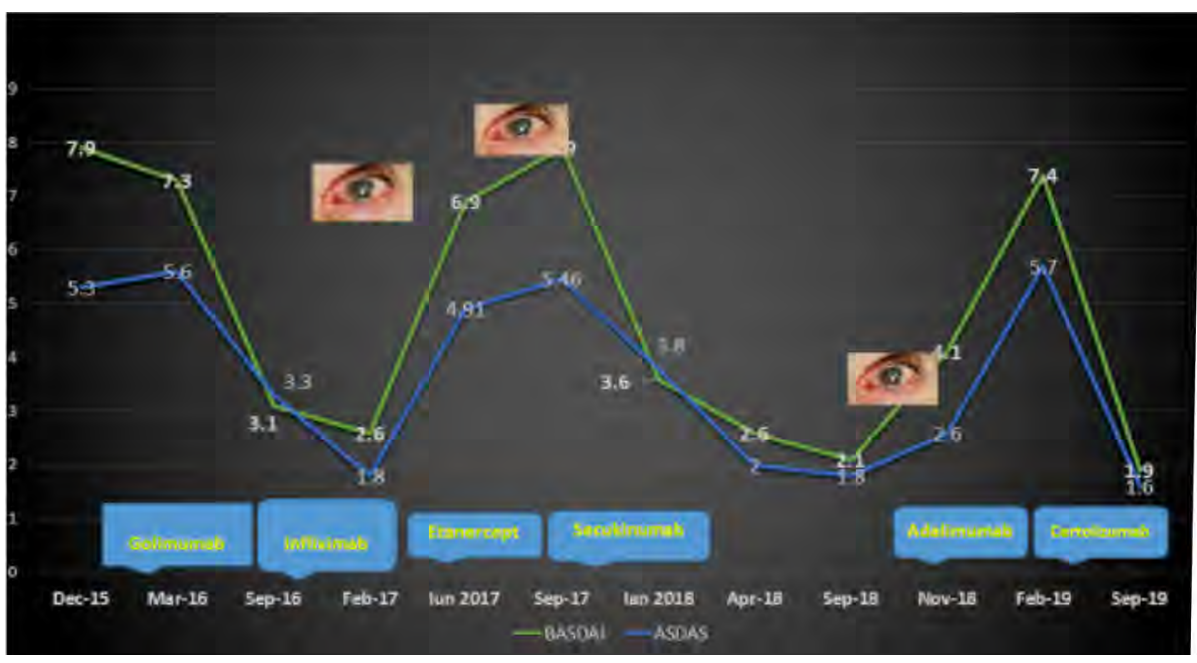

FIGURE 1. Evolution of ankylosing spondylitis activity according to BASDAI and ASDAS scores

There is no specific treatment for MGUS, patients need subsequent follow-up according to specific guidelines in order to detect progression to lymphoplasmacytic malignancies (LPMs) (2).

Data are limited regarding the influence of antiTNF on the progression of MGUS. Some case reports suggest that there may be an association between anti-TNF- $\alpha$ therapy and development of MGUS $(4,5)$. Still, large observational studies suggest the opposite. In a observational cohort of 444 patients with psoriatic arthropathy treated with biologics, the incidence and frequency of MGUS do not appear to increase relative to the general population (6). Moreover, the risk of progression to multiple myeloma in patients with MGUS treated with TNF-blockers does not seem to be increased compared with patients treated with conventional DMARDs (7).

Previous studies have evaluated the risk of incident cancer development among individuals exposed to anti-TNF therapy. In an early meta-analysis of

TABLE 1. Classification, diagnostic criteria, risk and pattern of progression of monoclonal gammopathies

\begin{tabular}{|c|c|c|c|}
\hline Subtype of MGUS & Diagnostic criteria & $\begin{array}{l}\text { Risk of } \\
\text { progression }\end{array}$ & Pattern of progression \\
\hline IgM MGUS & $\begin{array}{l}\text { All } 3 \text { criteria must be met: } \\
\text { - Serum IgM monoclonal protein }<3 \mathrm{gm} / \mathrm{dL} \\
\text { - Bone marrow lymphoplasmacytic infiltration }<10 \% * \\
\text { - No evidence of anemia, constitutional symptoms, } \\
\text { hyperviscosity, lymphadenopathy, or hepatosplenomegaly } \\
\text { that can be attributed to the underlying } \\
\text { lymphoproliferative disorder }\end{array}$ & $1 \%$ per year & $\begin{array}{l}\text { Waldenström } \\
\text { macroglobulinemia, AL } \\
\text { amyloidosis; rarely IgM } \\
\text { multiple myeloma }\end{array}$ \\
\hline Non-IgM MGUS & $\begin{array}{l}\text { All } 3 \text { criteria must be met: } \\
\text { - Serum monoclonal protein (non-lgM type) }<3 \mathrm{gm} / \mathrm{dL} \\
\text { - Clonal bone marrow plasma cells }<10 \% * \\
\text { - Absence of end-organ damage such as hypercalcemia, } \\
\text { renal insufficiency, anemia, and bone lesions (CRAB) that } \\
\text { can be attributed to the plasma cell proliferative disorder }\end{array}$ & $0.5 \%$ per year & $\begin{array}{l}\text { Multiple myeloma, } \\
\text { solitary plasmacytoma, AL } \\
\text { amyloidosis }\end{array}$ \\
\hline Light-chain MGUS & $\begin{array}{l}\text { All criteria must be met: } \\
\text { - Abnormal FLC ratio }(<0.26 \text { or }>1.65 \text { ) } \\
\text { - Increased level of involved light chain (increased } \mathrm{kLC} \\
\text { in patients with FLC ratio }>1.65 \text { and increased } \lambda \text { FLC in } \\
\text { patients with FLC ratio }<0.26 \text { ) } \\
\text { - No immunoglobulin heavy-chain expression on } \\
\text { immunofixation } \\
\text { - Absence of end-organ damage that can be attributed to } \\
\text { the plasma cell proliferative disorder } \\
\text { - Clonal bone marrow plasma cells }<10 \% * \\
\text { - Urinary monoclonal protein }<500 \mathrm{mg} \text { per } 24 \mathrm{~h}\end{array}$ & $0.3 \%$ per year & $\begin{array}{l}\text { Light-chain multiple myeloma } \\
\text { and } \mathrm{AL} \text { amyloidosis }\end{array}$ \\
\hline
\end{tabular}


data from randomized controlled trials utilizing anti-TNF therapy a dose-dependent increased risk of malignancy was demonstrated among individuals exposed to anti-TNF therapy (pooled OR 3.3, 95\% CI 1.2-9.1) (8). Subsequent observational registry studies failed to demonstrate an increased risk of incident cancer development compared to biologic-naive comparator populations (RR $1.00,95 \%$ CI $0.86-$ 1.15) (9). A meta-analysis demonstrated a pooled estimate for the risk of incident cancer from seven studies without a significantly increased risk of allsite malignancy (RR $0.95,95 \%$ CI 0.73-1.51) among patients with RA, psoriatic arthritis or ankylosing spondylitis (10). Furthermore, the risk of lymphoma was not increased (RR 1.11, 95\% CI 0.70-1.51) while were increased among individuals exposed to anti-TNF therapies in four studies and two studies, respectively (10).

A population based cohort study specifically investigated the risk of squamous cell and basal cell skin cancer in patients with rheumatoid arthritis naive to biologic drugs, in patients starting TNF inhibitor treatment, and in the general population(11). For basal cell cancer, the hazard ratio was $1.22(95 \% \mathrm{CI}$ 1.07 to 1.41 ) comparing biologics-naive rheumatoid arthritis patients with the general population and 1.14 (0.98 to 1.33 ) comparing TNF inhibitor treated patients with biologics-naive patients. For squamous cell cancer, the hazard ratio was 1.88 (1.74 to 2.03) comparing biologics-naive rheumatoid arthritis patients with the general population and 1.30 (1.10 to 1.55) comparing TNF inhibitors with biologics-naive patients. The authors concluded that a small to moderately increased risk of basal cell cancer was seen in biologics-naive rheumatoid arthritis patients, with no further effect of TNF inhibitors. Among people with a history of squamous cell or basal cell cancer, TNF inhibitors did not further increase risks (11).
Although the risk of incident cancer development among individuals exposed to anti-TNF therapy does not appear to be increased, when cancers develop, previous studies have demonstrated high rates of discontinuation of anti-TNF therapy (12). In a nationwide study evaluating cancer stage at diagnosis and risk of death following a diagnosis of cancer, no increased risk of death was demonstrated among individuals developing cancer while on anti-TNF therapy compared to a biologics-naïve control group (13). Current guidelines from The American College of Rheumatology (14) recommends starting or resuming biologic therapy for patients treated for a solid malignancy more than 5 years prior, while EULAR (15) and British Society (16) guidelines do not have specific time framings.

A recent meta-analysis and systematic review combining the incidence rates of cancer recurrence among individuals with a history of cancer and immunosuppression exposure was performed demonstrating similar results to include a pooled cancer incidence rate of 33.8 per 1,000 person-years among individuals receiving anti-TNF therapy and 37.5 per 1,000 person-years among individuals not receiving immunosuppressive therapy (17).

Another recent meta-analysis reviewed 92 trials, on 10 populations with inflammatory disorders (eight among individuals with a history of rheumatic disease and two among individuals with a history of inflammatory bowel disease) (18). The nine studies finally analysed included a total of 11,679 patients with a history of cancer, 3,707 exposed to antiTNF, the duration interval between original cancer diagnosis and initiation of anti-TNF therapy ranged from 1.2 years to 11.5 years. The pooled incidence rates of cancer development were $3.2 / 100$ patient-years (95\% CI 2.1-4.9) in the anti-TNF exposed group and 3.6/100 patient-years (95\% CI 2.3-5.6) in the control cohort. Among individuals with a prior history of

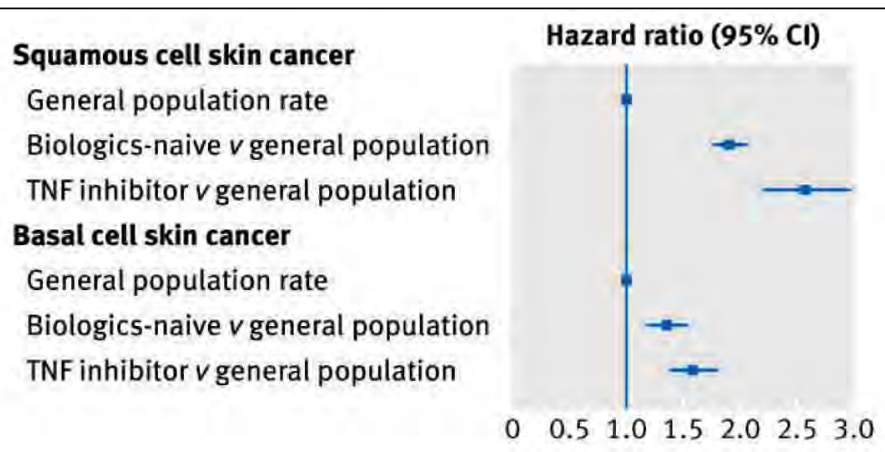

FIGURE 2. Hazard ratios (95\% Cl) for squamous cell cancer and basal cell cancer among biologics-naive patients with rheumatoid arthritis (RA) compared with matched general population comparators and among tumour necrosis factor (TNF) inhibitor treated patients with RA compared with general population comparators (11, reproduced with publisher's permission). 


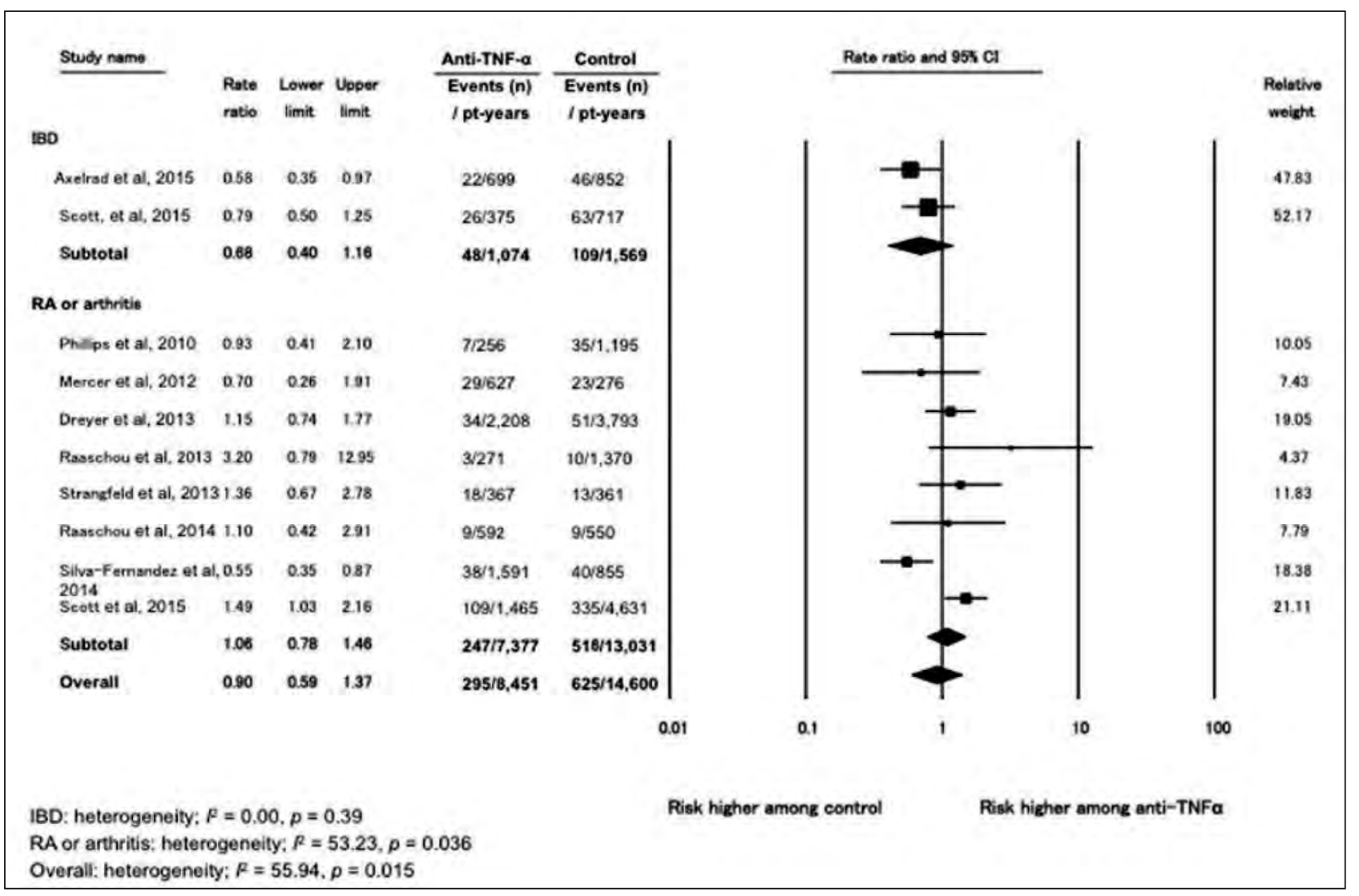

FIGURE 3. Risk of new or recurrent cancer development when exposed to anti-TNF therapy (reproduced with author's permission)

skin cancer, new or recurrent cancer was not increased $(0.89,95 \%$ CI $0.34-2.28)$. The authors concluded that the risk of new or recurrent cancer among individuals with a history of cancer exposed to anti-TNF therapy was not significantly different compared to control therapies. In terms of individual cancer types studied, there were no obvious differences in risk of new cancer development or cancer recurrence among individuals with a history of solid tumor malignancy, skin cancer, or when examining the subgroup excluding skin cancers (18).

Current opinions are still often divided on the actual role IL-17 plays in the pathophysiology of cancer, clearly demonstrating a need for more research in this area (19).

Patients of older age, with previous skin cancer or actinic damage, family history of skin cancers, concurrent or history of immunosuppressive therapies or therapies known to increase skin cancer risk (i.e., cyclosporine, phototherapy especially PUVA) are reported to be at increased risk of non-melanoma skin cancer (NMSC). It is possible that an increased reporting of NMSC with biologics may be attributable to increased detection of skin cancer rather than increased development; however, studies comparing NMSC in patients on biologics with control patients also demonstrated increased rates of NMSC (20).

\section{CONCLUSIONS}

As patients with immune mediated conditions continue to age, the lifetime risk of cancer progressively increases due to increases in life expectancy and increased age-specific rates of various cancers. Combined with the improved prognosis of individuals with a history of cancer, rheumatologists increasingly will be faced with the challenge of managing patients with a history of cancer. Care should be taken with a multi-disciplinary approach to adequately discuss the risk of individual disease recurrence and the known risks and benefits of anti-TNF therapy for modifying clinical disease activity. Further large scale observational studies are required to assess clinical factors associated with cancer recurrence in the variety of cancer subtypes. 


\section{REFERENCES}

1. Deodhar A, Mease PJ, Mclnnes IB et al. Long-term safety of secukinumab in patients with moderate-to-severe plaque psoriasis, psoriatic arthritis, and ankylosing spondylitis: integrated pooled clinical trial and post-marketing surveillance data. Arthritis Res Ther. $2019-2 ; 21(1): 111$

2. Go SR, Rajkumar SV How I manage monoclonal gammopathy of undetermined significance Blood 2018 131:163-173.

3. Ding T, Ledingham J, Luqmani R et al. BSR and BHPR rheumatoid arthritis guidelines on safety of anti-TNF therapies. Rheumatology (Oxford). 2010 Nov;49(11):2217-9.

4. Smale SW, Lawson TM. Monoclonal gammopathy of undetermined significance and anti-TNF-alpha treatment. Scand J Rheumatol. 2007;36(5):405-6.

5. Prignano F, Pescitelli L, Ricceri F et al. Development of MGUS in psoriatic patients: A possible undiagnosed event during anti-TNF-atreatment. J Eur Acad Dermatol Venereol. 2012;26(11):1444-8.

6. Liegeon A., Mahe E, Begon E et al. Development of monoclonal gammopathy under biotherapy in psoriasis: A French multicenter retrospective study. Eur J Dermatol 2016; 26:75-81.

7. Lernia VD, Ficarelli E, Lallas A et al. Frequency of monoclonal gammopathy in psoriatic patients receiving anti-TNFtherapy compared with patient staking conventional drugs: A cross-sectional study. European Academy of Dermatology and Venereology 2015; 29(11):2262-3.

8. Bongartz T, Sutton AJ, Sweeting MJ et al. Anti-TNF antibody therapy in rheumatoid arthritis and the risk of serious infections and malignancies: Systematic review and meta-analysis of rare harmful effects in randomized controlled trials. JAMA. 2006; 295(19):2275-85.

9. Askling J, van Vollenhoven RF, Granath F et al. Cancer risk in patients with rheumatoid arthritis treated with anti-tumor necrosis factor alpha therapies: Does the risk change with the time since start of treatment? Arthritis Rheum. 2009;60(11):3180-9.

10. Mariette X, Matucci-Cerinic M, Pavelka K et al. Malignancies associated with tumour necrosis factor inhibitors in registries and prospective observational studies: A systematic review and metaanalysis. Ann Rheum Dis. 2011;70(11):1895-904.

11. Raaschou P, Simard JF, Asker Hagelberg C. Rheumatoid arthritis, anti-tumour necrosis factor treatment, and risk of squamous cell and basal cell skin cancer: Cohort study based on nationwide prospectively recorded data from Sweden. BMJ. 2016 Jan 28; 352:i262.

12. Guerra I, Algaba A, Quintanilla E et al. Management and course of inflammatory bowel disease patients with associated cancer. J Crohns Colitis. 2014 Feb;8(1):S49. Incidence, management, and course of cancer in patients with inflammatory bowel disease.

13. Raaschou P, Simard JF, Neovius M et al. Does cancer that occurs during or after anti-tumor necrosis factor therapy have a worse prognosis? A national assessment of overall and site-specific cancer survival in rheumatoid arthritis patients treated with biologic agents. Arthritis Rheum. 2011; 63(7):1812-22.

14. Singh J.A., Saag K.G., Bridges, SL et al. 2015 American College of Rheumatology Guideline for the Treatment of Rheumatoid Arthritis. Arthritis Care \& Research 2015, 68:1-25.

15. Holroyd CR, Seth R, Bukhari M et al. The British Society for Rheumatology biologic DMARD safety guidelines in inflammatory arthritis - Executive summary. Rheumatology 2019;58(2):220-226

16. Smolen JS, Landewé R, Bijlsma J et al. EULAR recommendations for the management of rheumatoid arthritis with synthetic and biological disease-modifying antirheumatic drugs: 2016 update. Ann Rheum Dis. 2017 Jun;76(6):960-977.

17. Shelton E, Laharie D, Scott Fl et al. Cancer Recurrence Following Immune-Suppressive Therapies in Patients With ImmuneMediated Diseases: A Systematic Review and Meta-analysis. Gastroenterology. 2016 Jul;151(1):97-109.e4.

18. Micic D, Komaki Y, Alavanja A, Rubin DT Risk of Cancer Recurrence Among Individuals Exposed to Antitumor Necrosis Factor Therapy: A Systematic Review and MetaAnalysis of Observational Studies. J Clin Gastroenterol. 2019 Jan;53(1):e1-e11.

19. Yang $\mathrm{B}$, Kang $\mathrm{H}$, Fung $\mathrm{A}$, Zhao $\mathrm{H}$ et al. The role of interleukin 17 in tumour proliferation, angiogenesis, and metastasis. Mediators Inflamm. 2014;2014:623759.

20. https://www.ema.europa.eu/en/documents/rmp-summary/cosentyxepar-risk-management-plan-summary_en.pdf.

Conflict of interest: none declared Financial support: none declared 